\title{
Comparison of the Complications in Vertical Rectus Abdominis Musculocutaneous Flap with Non-Reconstructed Cases after Pelvic Exenteration
}

\author{
Heechang Jeon, Eul Sik Yoon, Hi-Jin You, Hyon-Surk Kim, Byung-Il Lee, Seung Ha Park \\ Department of Plastic and Reconstructive Surgery, Korea University Anam Hospital, Korea University College of Medicine, Seoul, Korea
}

Background Perineal reconstruction following pelvic exenteration is a challenging area in plastic surgery. Its advantages include preventing complications by obliterating the pelvic dead space and minimizing the scar by using the previous abdominal incision and a vertical rectus abdominis musculocutaneous (VRAM) flap. However, only a few studies have compared the complications and the outcomes following pelvic exenteration between cases with and without a VRAM flap. In this study, we aimed to compare the complications and the outcomes following pelvic exenteration with or without VRAM flap coverage.

Methods We retrospectively reviewed the cases of nine patients for whom transpelvic VRAM flaps were created following pelvic exenteration due to pelvic malignancy. The complications and outcomes in these patients were compared with those of another nine patients who did not undergo such reconstruction.

Results Flap reconstruction was successful in eight cases, with minor complications such as wound infection and dehiscence. In all cases in the reconstructed group $(n=9)$, structural integrity was maintained and major complications including bowel obstruction and infection were prevented by obliterating the pelvic dead space. In contrast, in the control group $(n=9)$, peritonitis and bowel obstruction occurred in 1 case (11\%).

Conclusions Despite the possibility of flap failure and minor complications, a VRAM flap can result in adequate perineal reconstruction to prevent major complications of pelvic exenteration.

Keywords Pelvic exenteration / Rectus abdominis / Musculocutaneous flap

\author{
Correspondence: Eul Sik Yoon \\ Department of Plastic and \\ Reconstructive Surgery, Korea \\ University Anam Hospital, Korea \\ University College of Medicine, \\ 73 Inchon-ro, Seongbuk-gu, \\ Seoul 136-705, Korea \\ Tel: +82-2-920-5368 \\ Fax: +82-2-922-7437 \\ E-mail: yesanam2@korea.ac.kr
}

No potential conflict of interest relevant to this article was reported.

\section{INTRODUCTION}

Although perineal reconstruction following pelvic exenteration is a challenge for plastic surgeons, it has not been performed as often as head and neck reconstruction following malignancy due to the high rate of donor morbidity and the fact that reconstruction is not indicated in all cases. Nevertheless, perineal recon- struction has its advantages in preventing complications of pelvic exenteration by obliterating the pelvic dead space; it also minimizes the donor scar by using the previous abdominal incision and a vertical rectus abdominis musculocutaneous (VRAM) flap. However, few studies have compared the complications and the outcomes following pelvic exenteration between cases with and without a VRAM flap. This study aimed to compare the 
complications and the outcomes following pelvic exenteration with or without VRAM flap coverage.

\section{METHODS}

All patients who underwent pelvic exenteration and immediate reconstruction with a pedicled VRAM flap $(\mathrm{n}=9)$ between 2011 and 2013 at our center were retrospectively reviewed. We reviewed the patient, tumor, and treatment characteristics; surgical outcomes; and postoperative donor- and recipient-site complications. We also collected information on patients who underwent pelvic exenteration without any reconstruction surgery $(n=9)$ between 2011 and 2013 to serve as a control group for comparison with the VRAM group. Patients with incomplete data on postoperative complications were excluded from this study.

The surgery was performed under general anesthesia in all cases, and the patient was placed in the lithotomy position. Pelvic exenteration was first done according to the range of the tumor. Using a hand-held Doppler ultrasound device, one can easily identify the perforators. Skin paddle dimensions ranged from $5 \mathrm{~cm} \times 12 \mathrm{~cm}$ to $8 \mathrm{~cm} \times 13 \mathrm{~cm}$, with the longest axis oriented vertically. The dimensions of the flap were tailored to the size of the perineal defect. The flap was elevated vertically from the costal margin to the level of the inguinal fold, and the longitudinal incision line was made to include the pre-existing laparotomy incision (Fig. 1). Subcutaneous dissection was carried out to the level of the rectus sheath where the lateral border was incised. The rectus muscle was then elevated off the posterior sheath. Thereafter, the deep inferior epigastric vessels were identified. At this point, the pedicle was mobilized to its origin and separated from all surrounding tissue attachments to prevent kinking or compression. A tunnel was then made beneath the pubic ramus, and the flap was delivered into the perineum. The muscle was divided from the skin island superiorly by using electrocautery, and ligation of the superior epigastric vessels was performed with vessel clips. The flap was rotated medially and transposed onto the perineal defect to obliterate the maximum amount of dead space possible and to prevent tension. A suction drainage system was placed in the pelvic space. The skin paddle of the flap was then inset into the defect margin.

Postoperatively, the flap was monitored by conventional techniques, which included Doppler ultrasonography. The patients were placed on strict bed rest on an air mattress for at least 96 hours. Postoperative positioning was in thigh abduction with a pillow placed under the sacrum to limit pressure on the perineal wound during hospitalization. Adequate perioperative prophylactic antibiotic therapy was administered for 7 days, and intravenous prostaglandin was given to all patients until 5 days post-

\section{Fig. 1. A design of the incision line}

A longitudinal incision line should include the pre-existing laparotomy incision.

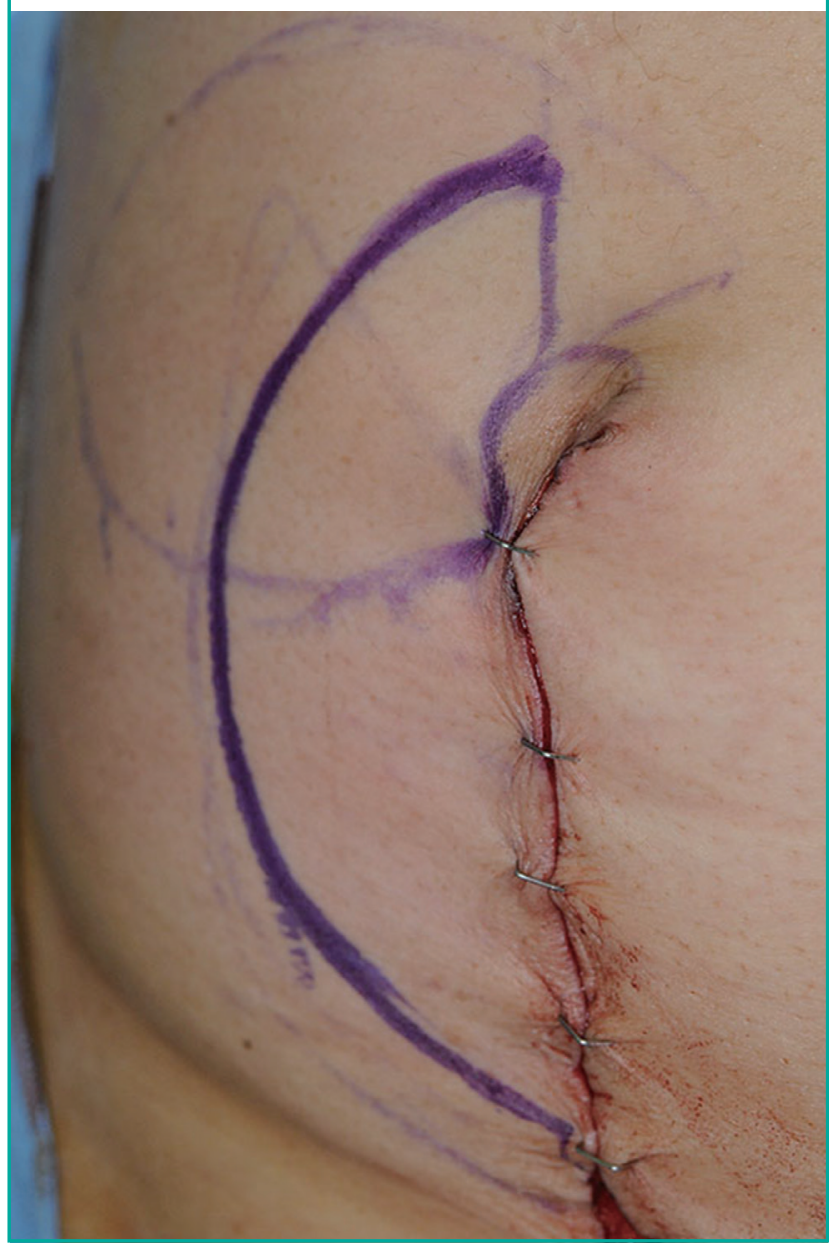

operatively.

For the comparison of complications between the two groups, we applied the classification of surgical complications first proposed by Clavien et al. [1] According to the relevant criteria, each complication was classified into one or more of six categories. The point value for each complication was compared between the groups as described by the comprehensive complication index (CCI) [2].

\section{RESULTS}

Among the 9 patients in the VRAM flap group, one showed mild dehiscence (11\%) and another showed wound infection (11\%). The overall characteristics of the patients are listed in Table 1. In the patient with the wound infection, the flap was originally repositioned at its primary site (abdomen), since there was no need for reconstruction after flap elevation. After repositioning, signs of infection followed, and for the prevention of peritonitis, 
flap excision was performed. Peritoneal complications that frequently occur when direct closure is performed, including bowel obstruction, peritonitis, perineal oozing, or seroma formation, did not occur in the patients who underwent reconstruction. Other significant complications such as total necrosis or hematoma also did not occur, and the patients reported being generally satisfied with their appearance.

In contrast, in the control group, peritonitis and bowel obstruction occurred in 1 case (11\%) (Table 2).

Each complication was classified according to the Clavien-Dindo classification, and the sum of the CCI values was compared between the groups. The average CCI values were 12 and 16.2 in the reconstructed group and the control group, respectively.

\section{Case 1}

A 59-year-old woman with adenocarcinoma of the rectum underwent immediate perineum and pelvic cavity reconstruction

Table 1. Demographic characteristics of the patients
\begin{tabular}{|lcccll|}
\hline No. & Age (yr) & Location & Histology & Resection & Complications \\
\hline 1 & 59 & Rectum & AC & Posterior & Mild dehiscence \\
2 & 65 & Cervix & SCC & Anterior & None \\
3 & 55 & Cervix & SCC & Anterior & Partial necrosis \\
4 & 71 & Cervix & SCC & Anterior & None \\
5 & 60 & Cervix & SCC & Anterior & None \\
6 & 70 & Cervix & SCC & Anterior & None \\
7 & 62 & Cervix & SCC & Anterior & None \\
8 & 61 & Cervix & SCC & Anterior & None \\
9 & 65 & Rectum & AC & Posterior & None \\
\hline AC, adenocarcinoma; SCC, squamous cell carcinoma. & \\
\hline
\end{tabular}

after posterior pelvic exenteration. A $7 \mathrm{~cm} \times 12 \mathrm{~cm}$ right-pedicled VRAM flap was elevated and inset in the pelvic floor and the perineal wound. Minor wound dehiscence in the gluteal fold developed on day 7 and was treated with debridement and delayed repair. There were no significant donor-site complications. At the 2-month follow-up examination, the integrity of the perineum was maintained, and the patient was satisfied with the result (Fig. 2).

\section{Case 2}

A 65-year-old woman with squamous cell carcinoma of the cervix with bladder, vulva, and rectum wall invasion underwent immediate perineal reconstruction after anterior pelvic exenteration. An $8 \mathrm{~cm} \times 10 \mathrm{~cm}$ left-pedicled VRAM flap was used for pelvic wound closure and obliteration of the pelvic dead space. The postoperative course was stable without any significant

\section{Table 2. Outline of postoperative complications}

\begin{tabular}{|lcc|}
\hline Characteristic & $\begin{array}{c}\text { Pelvic exenteration } \\
\text { with VRAM flap } \\
\text { coverage }\end{array}$ & $\begin{array}{c}\text { Pelvic exenteration } \\
\text { without } \\
\text { reconstruction }\end{array}$ \\
\hline No. of patients $(\mathrm{n})$ & 9 & 9 \\
Dehiscence & $1(11)$ & $0(0)$ \\
Wound infection & $1(11)$ & $0(0)$ \\
Partial flap necrosis & $1(11)$ & $0(0)$ \\
Total flap necrosis & $0(0)$ & $0(0)$ \\
Seroma & $0(0)$ & $0(0)$ \\
Peritonitis & $0(0)$ & $1(11)$ \\
Bowel obstruction & $0(0)$ & $1(11)$ \\
\hline Values are presented as number (\%). & \\
VRAM, vertical rectus abdominis musculocutaneous. & \\
\hline
\end{tabular}

Fig. 2. A case of posterior exenteration

(A) Intraoperative view of flap harvest. (B) Immediate postoperative photograph of the perineum. (C) Abdominal donor site after healing.
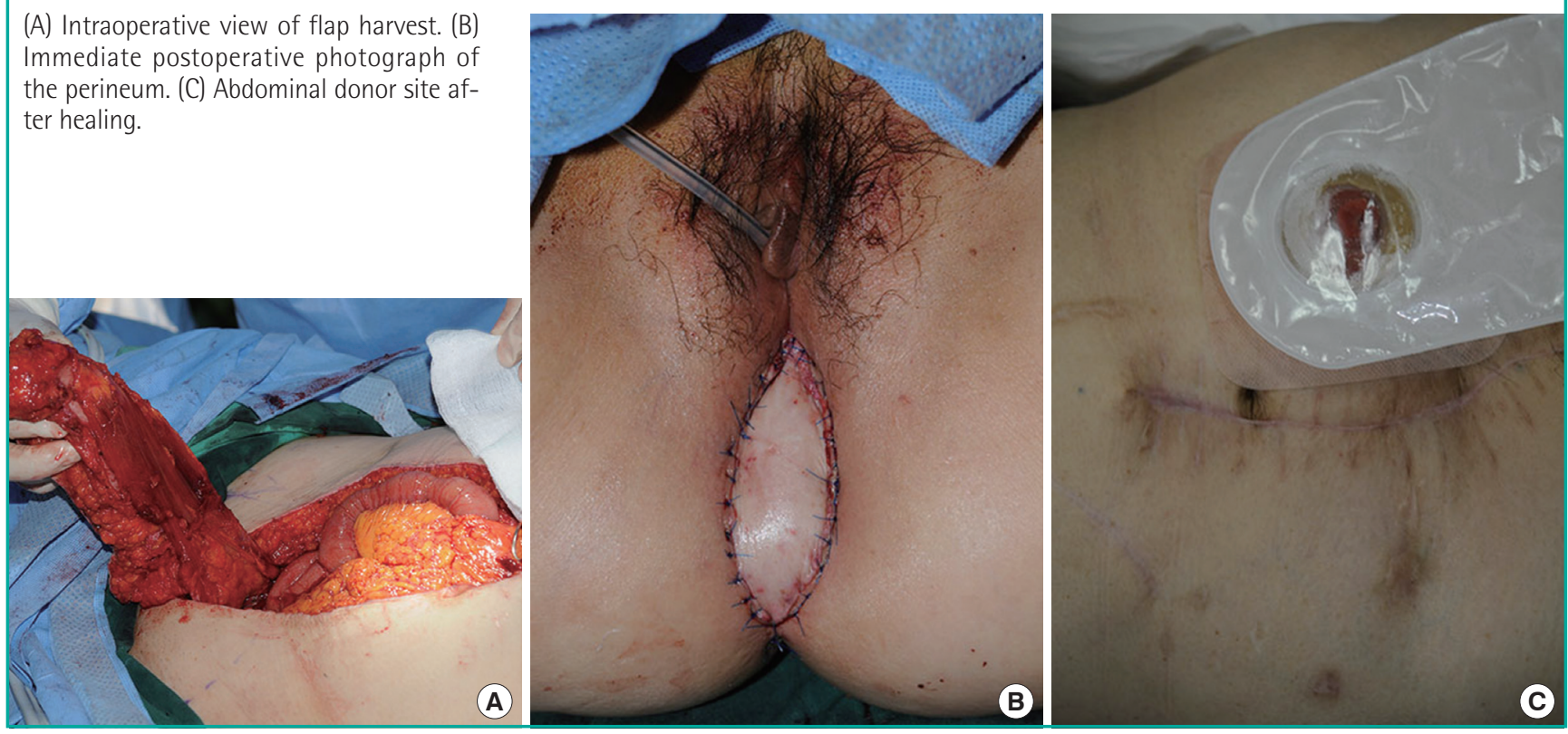
complications. At the 1-month follow-up examination, the status of the flap was acceptable and the patient was satisfied with the result (Fig. 3).

\section{Case 3}

A 55-year-old woman with recurrent squamous cell carcinoma of the cervix had planned to undergo pelvic exenteration and im- mediate reconstruction. At the beginning of the surgery, VRAM flap elevation was done prior to performing pelvic exenteration. However, after the procedure, there appeared to be sufficient perineal skin and therefore, no need for flap reconstruction. The flap was therefore relocated to the original site. Excessive tension was noted during the closure of the fascia performed by gynecologists, and partial necrosis of the flap was observed on post-

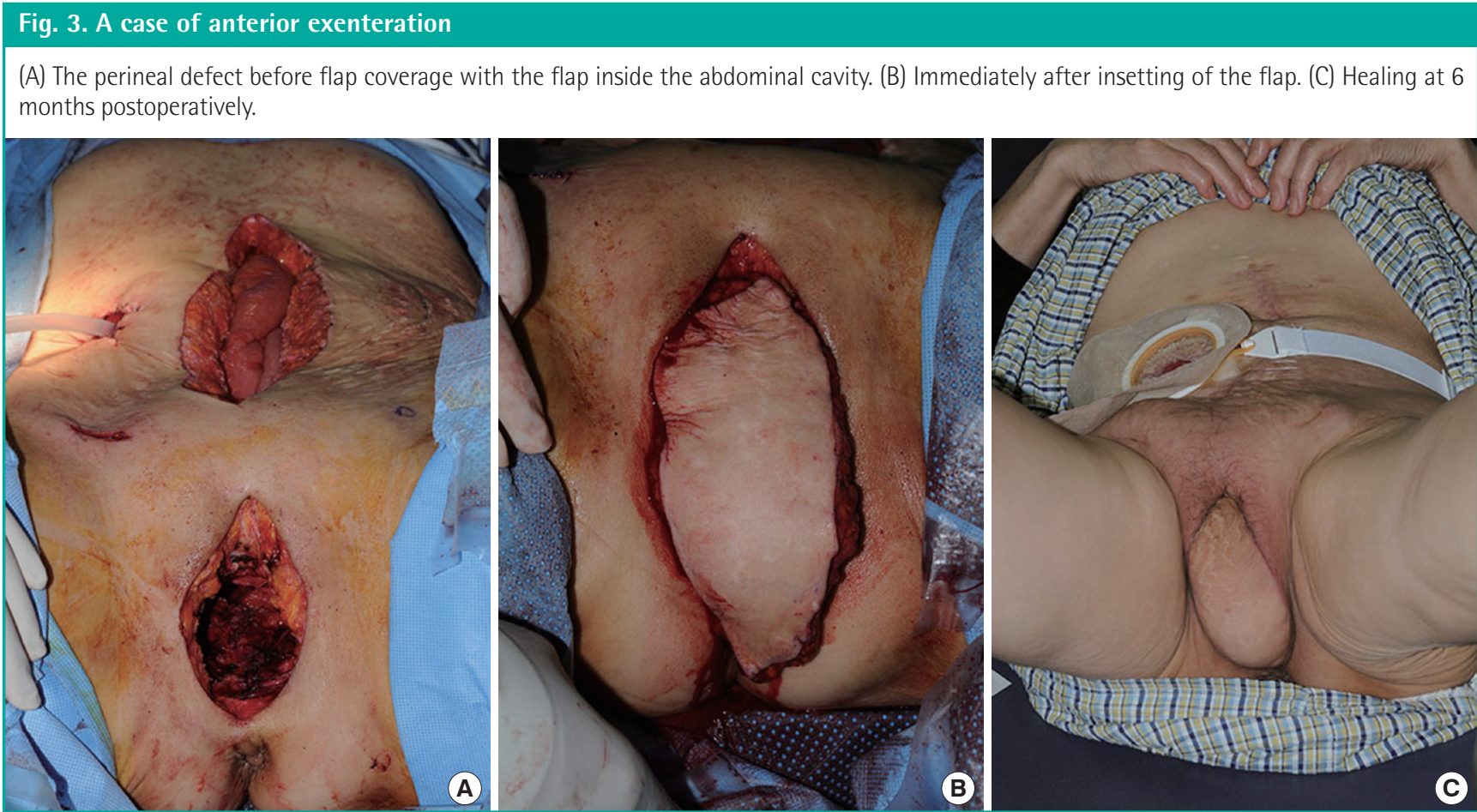

Fig. 4. A case with flap removal and dehiscence

(A) The preoperative diagram on the left hemiabdomen. (B) The intraoperative view of the deep inferior epigastric pedicle. The flap was relocated to the abdomen donor site. (C) After flap removal due to excessive tension, mild wound dehiscence occurred on the lower abdomen.
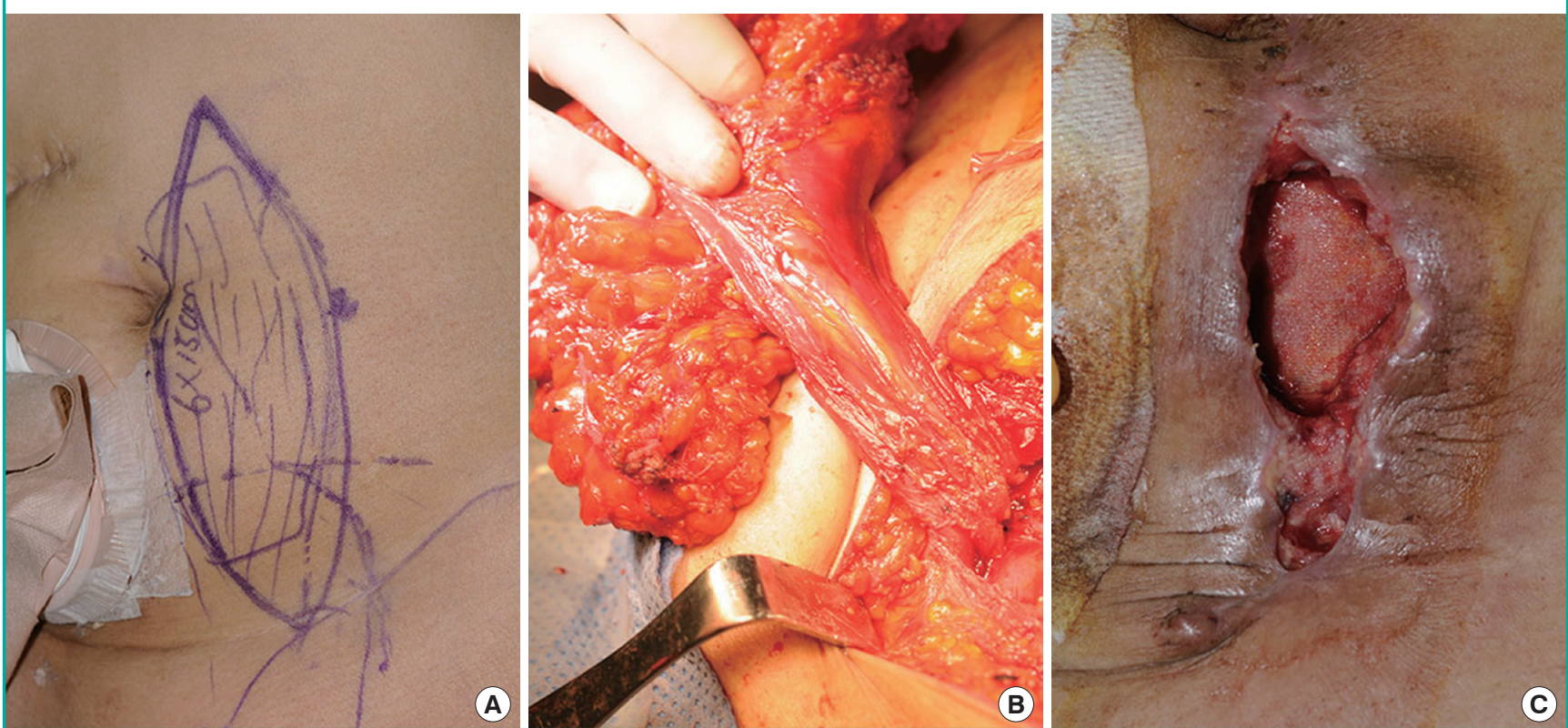
operative day 2. Pus and other infection signs occurred the following day. For the prevention of pan-peritonitis resulting from an infection of the surgical site, excision of the VRAM flap and anterior rectus fascia reconstruction using an artificial mesh was performed on postoperative day 4 . Thereafter, wound dehiscence and sloughing of the lower abdomen were observed, but healing was good after the debridement (Fig. 4).

\section{DISCUSSION}

Pelvic exenteration is an operation performed for the treatment of pelvic organ malignancy. Despite the destructive properties of the operation, there have been only limited attempts to perform immediate postoperative reconstruction. This is primarily due to the prolonged operating time required and the fact that primary closure is possible in most cases. After pelvic exenteration, 38\% of the patients had more than one life-threatening surgical complication such as sepsis, peritonitis, bowel obstruction, and incarceration, and $29 \%$ of the patients needed additional surgical treatment such as exploratory laparotomy, bowel resection, and delayed repair [3]. In other published studies, mortality rates as high as $40 \%$ to $60 \%$ have been reported for reoperation to correct intestinal and genitourinary complications following exenteration [4]. This is related to the large, non-collapsible dead space and the irradiated, poorly vascularized field (Fig. 5). Therefore, considering the frequency of complications after pelvic exenteration, immediate steps to structurally prevent these complications can be a cost-effective solution.

Pelvic prolapse of the small bowel can cause bowel adhesion, obstruction, and fistula formation. Moreover, it can influence the recurrence of the cancer, as it limits the range and the dose

Fig. 5. A case of posterior pelvic exenteration without any reconstruction

Mild increased tension of the perineal skin flap was seen at 3 days postoperatively.

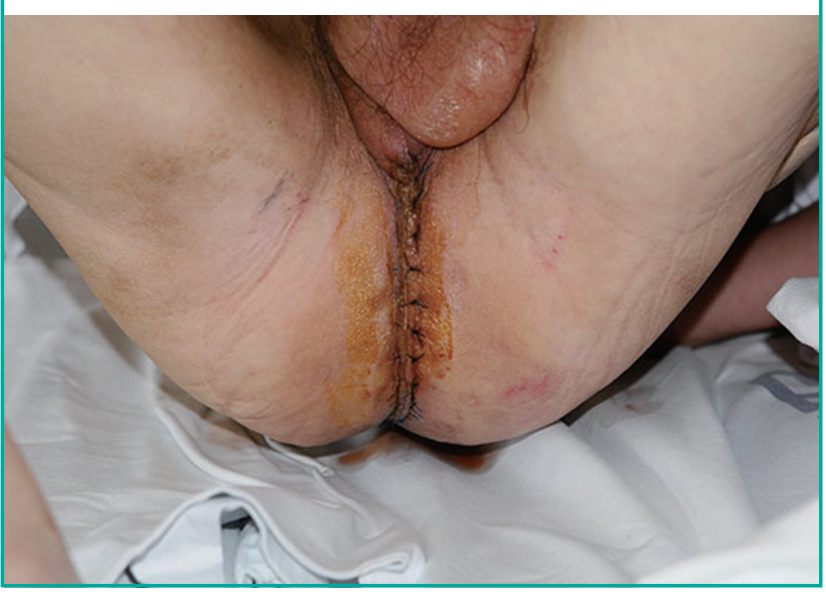

of the radiation treatment, thereby reducing the effectiveness of the treatment. Dead space in the pelvis is the greatest source of postoperative morbidity in those who have undergone exenteration, particularly following treatment with radiation. An approximation of local tissues and primary wound healing is often not possible because of the lack of mobility of the pelvis and soft tissues. Even if closure is achieved without tension, the irradiated tissue may not heal. Delayed perineal wound healing adds to patient discomfort, increased hospital stay, and the possibility of additional operative procedures [5]. To prevent these complications, a tension-free coverage of dead space with a well-vascularized tissue is required. The well-vascularized tissue is metabolically active and therefore facilitates the delivery of oxygen, products of the immune system, and systemically administered antibiotics and chemotherapeutics to the cells.

Furthermore, the creation of an abdominopelvic partition prevents small bowel prolapse, which can lead to radiation enteritis, bowel obstruction, or fistula formation [5]. The use of silicone implants and absorbable meshes as abdominopelvic partitions has been reported. However, procedures using artificial materials have several morbidities such as inflammation and capsule formation [6]. Alternatively, an autologous tissue can be used. There are several options, including omentum and myocutaneous flaps. The omentum can reach into the pelvis, bringing vascularized tissue supplied by the gastroepiploic artery. However, it lacks tensile strength and can result in herniation of the bowel through the perineal defect. Although it can cover the raw surface of the pelvis, it does not effectively exclude the bowel; therefore, morbidity remains high [3]. A myocutaneous flap is an ideal option, as it contains a mass of vascular tissue and facilitates wound healing by providing a greater blood supply [7]. While skin necrosis can occur, the muscle is often viable and granulates like normal tissue with wound closure in weeks compared with the months needed in situations where no myocutaneous flap was brought into the wound.

Since McCraw et al. [8] introduced the use of the gracilis musculocutaneous pedicled flap for the reconstruction of the pelvis and the perineum, it has been the workhorse for pelvic reconstructive surgeons. However, experience has shown that the viability of the flap is unreliable, particularly in the distal third; in large pelvic defects, the muscle often lacks sufficient length and bulk and does not carry a skin paddle well. Although the morbidity of exenterations is lessened with the use of a gracilis flap, it remains high. Partial or total flap loss may occur in $10 \%$ to $24 \%$ of the patients. As many as $37 \%$ of the patients require a subsequent procedure or experience delayed perineal wound healing, particularly if the patient has undergone irradiation [9].

The rectus abdominis myocutaneous flap was first established 
by Mathes and Bostwick [10]. Its use has since been extended to a wide variety of indications related to the anterior trunk and groin region. The vascular supply of the rectus muscle is from both the inferior and the superior epigastric vessels. A transpelvic modification of this flap was originally described in 1984 by Shukla and Hughes [11]. It provides more rapid and suitable healing of the perineal wound postexenteration, and prevents several postoperative complications.

Although there are other several perforator flap options that can cover a pelvic defect, a myocutaneous flap should be the first preference because of the need for filling the potential dead space in the pelvic cavity. Perforator flaps based on arteries such as the pudendal artery, superior and inferior gluteal artery, and perineal artery can be applied after pelvic exenteration; they are often limited to small-to-moderate skin defects or vaginal reconstruction [12].

A limitation of this study was its small sample size, and for this reason, we could not compare the data by using a conventional method of statistical analysis. Instead, we used the method of classifying surgical complications and the CCI to compare the severity of complications between the two study groups objectively.

As our case reports show, perineal reconstruction using a VRAM flap is an effective surgical procedure for both anterior and posterior pelvic exenteration. Further, it maintains the physical integrity of the patient and prevents major postoperative complications such as bowel obstruction and infection by obliterating the dead space of the pelvic cavity. Perineal reconstruction after pelvic exenteration using a VRAM flap can result in a clinical outcome superior to that of the group without reconstruction in addition to the prevention of major complications. However, as shown in one case, partial necrosis can occur due to excessive tension at closure. To obtain stronger evidence to support the use of this procedure, there should be additional research examining a larger number of cases.

\section{REFERENCES}

1. Clavien PA, Barkun J, de Oliveira ML, et al. The ClavienDindo classification of surgical complications: five-year experience. Ann Surg 2009;2009:187-96.

2. Slankamenac K, Graf R, Barkun J, et al. The comprehensive complication index: a novel continuous scale to measure surgical morbidity. Ann Surg 2013;258.

3. Soper JT, Berchuck A, Creasman WT, et al. Pelvic exenteration: factors associated with major surgical morbidity. Gynecol Oncol 1989;35:93-8.

4. Orr JW Jr, Shingleton HM, Hatch KD, et al. Gastrointestinal complications associated with pelvic exenteration. Am J Obstet Gynecol 1983;145:325-32.

5. Jain AK, DeFranzo AJ, Marks MW, et al. Reconstruction of pelvic exenterative wounds with transpelvic rectus abdominis flaps: a case series. Ann Plast Surg 1997;38:115-22.

6. Ball AB, Cassoni A, Watkins RM, et al. Silicone implant to prevent visceral damage during adjuvant radiotherapy for retroperitoneal sarcoma. Br J Radiol 1990;63:346-8.

7. Mathes SJ, Feng LJ, Hunt TK. Coverage of the infected wound. Ann Surg 1983;198:420-9.

8. McCraw JB, Massey FM, Shanklin KD, et al. Vaginal reconstruction with gracilis myocutaneous flaps. Plast Reconstr Surg 1976;58:176-83.

9. Heath PM, Woods JE, Podratz KC, et al. Gracilis myocutaneous vaginal reconstruction. Mayo Clin Proc 1984;59:21-4.

10. Mathes SJ, Bostwick J 3rd. A rectus abdominis myocutaneous flap to reconstruct abdominal wall defects. Br J Plast Surg 1977;30:282-3.

11. Shukla HS, Hughes LE. The rectus abdominis flap for perineal wounds. Ann R Coll Surg Engl 1984;66:337-9.

12. Kim JT, Ho SY, Hwang JH, et al. Perineal perforator-based island flaps: the next frontier in perineal reconstruction. Plast Reconstr Surg 2014;133:683e-687e. 